This item was submitted to Loughborough's Research Repository by the author.

Items in Figshare are protected by copyright, with all rights reserved, unless otherwise indicated.

\title{
Surface texturing for enhanced tribological performance
}

PLEASE CITE THE PUBLISHED VERSION

https://www.worldscientific.com/worldscibooks/10.1142/q0152

PUBLISHER

World Scientific Europe Ltd

VERSION

AM (Accepted Manuscript)

PUBLISHER STATEMENT

RAHMANI, R., 2018. Surface texturing for enhanced tribological performance. IN: Gohar, R. and Rahnejat, H. (eds.) Fundamentals of Tribology, 3rd ed. Copyright @ 2019 with permission from World Scientific Publishing Co. Pte. Ltd.

\section{LICENCE}

CC BY-NC-ND 4.0

\section{REPOSITORY RECORD}

Rahmani, Ramin. 2019. "Surface Texturing for Enhanced Tribological Performance". figshare. https://hdl.handle.net/2134/34462. 


\section{Chapter 15: Surface Texturing for Enhanced Tribological Performance}

\section{1- Introduction}

Along with the pursuit of coatings to enhance tribological performance and development of formulated lubricants with appropriate additive packages, surface texturing techniques have also been developed to guard against direct interaction of contacting surfaces. We have already shown (chapter 1) that mixed and boundary regimes of lubrication occur as the result of any direct interaction of rough surfaces. This increases the generated friction and reduces the system efficiency, as well as giving rise to wear and other forms of surface damage. The surface textures act as small reservoirs of lubricant, under conditions which retention of a coherent film of lubricant would not be expected, such as in cessation of rolling and/or sliding motion or in the presence of high contact loads. In chapter 12 we show the role of surface textures in piston-cylinder system and its beneficial effect in terms of reduced friction, thus power loss. In chapter 12 we provided results of some numerical analysis for textured surfaces as well as some experimental benefit of their effect in terms of reduction of power loss. However, the numerical predictive approach is quite complex and leads to long computation times. Furthermore, not everyone has access to such complex numerical models. Therefore, here we provide some representative analytical methods.

Surface texturing is an attempt to control the randomness of the inherent surface roughness. We note that lubricant fills the valleys between asperity peaks of rough contiguous surfaces and aids lubrication. However, this occurs in a rather random manner as the result of manufactured surfaces (chapter 2) and not in a controlled manner. Artificially-created texture features are normally in an intermediate scale between conjunctional geometrical gap and the level of surface roughness. In other words, as the texture features become smaller they merge into the overall surface topography, and as they increase in size they become part of the conjunctional geometry. Therefore, there are two elements to be considered when dealing with textured surfaces. These are the cumulative action of the surface features as an ensemble and their individual effects as micro-bearings. For instance, in a partially textured surface, the build-up of pressure can be viewed against the background of the overall conjunctional pressures, whereas in a fully textured contact, the rise in pressure at each individual surface feature can also occur. Textures cause pressure perturbations similar to the microelastohydrodynamic effect described in chapter 14. This point is made clearer in the rest of this section.

The main mechanism underlying the enhanced tribological performance (reduced friction and wear) with textured surfaces can be attributed, inter alia, to factors such as their ability in promoting micro-wedges and entrapping a reservoir of lubricant.

Modifying the structure of surfaces to gain improved mechanical and physical properties with operational performance in mind has been known in the automotive industry for quite a while 
R. Gohar and H. Rahnejat, Fundamental of Tribology, $3^{\text {rd }}$ Ed., World Scientific Publishing Europe Ltd., Singapore, 2018

(e.g. introduction of the cross-hatched cylinder liners (see Figure 15.1). It is believed that the cross-hatched liner surfaces are particularly good in retaining and directing the supply of lubricant to the piston and rings' contacts with the cylinder liner.

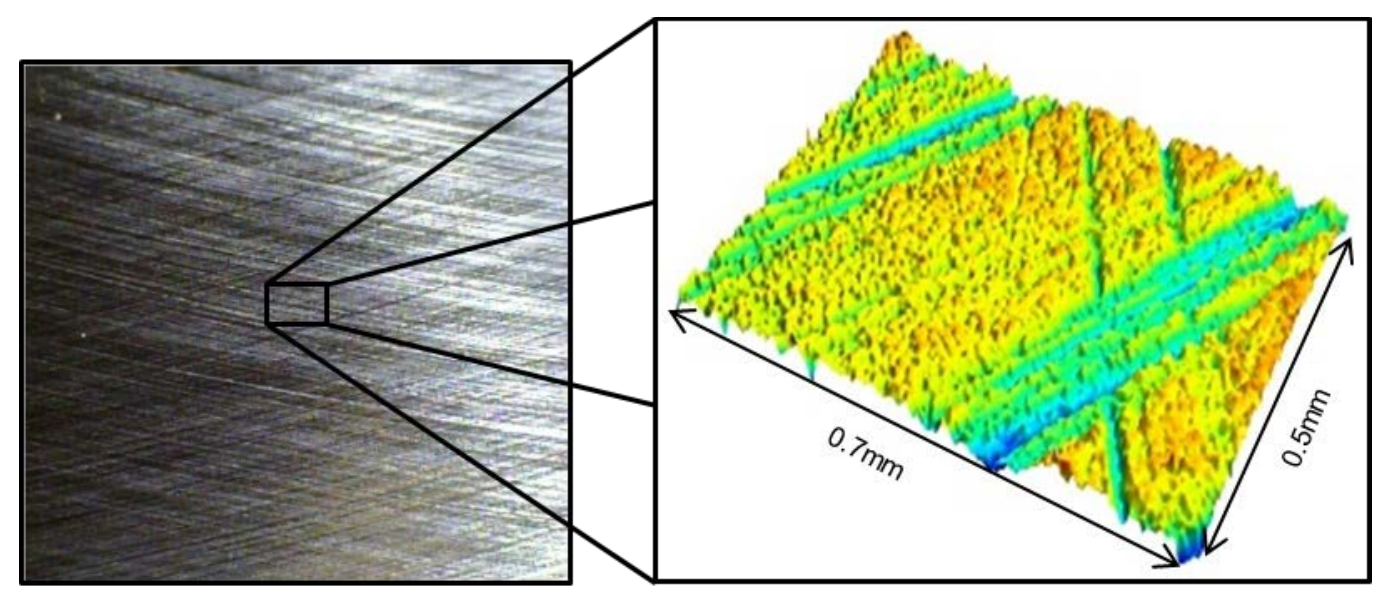

Figure 15.1: Topography of a steel cross-hatched liner

However, the idea of using textured surfaces relates to the use of determined (distinctive) features which are produced in specific shapes, form and distribution in particular locations in a contact.

These features can be in the form of grooves, corrugations, troughs, chevrons, dimples, pockets or pores. Figures 15.2- 15.4 illustrate various examples of textured surfaces.

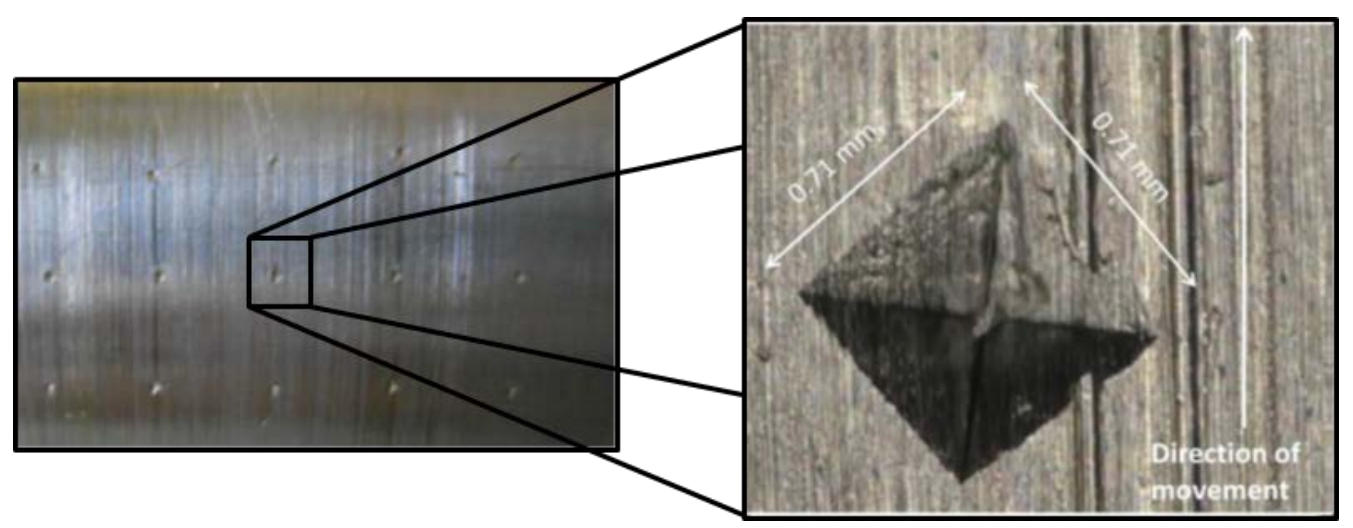

Figure 15.2: Pyramid shape dimples produced on a journal bearing shaft using a micro-indenter [1] 
R. Gohar and H. Rahnejat, Fundamental of Tribology, $3^{\text {rd }}$ Ed., World Scientific Publishing Europe Ltd., Singapore, 2018
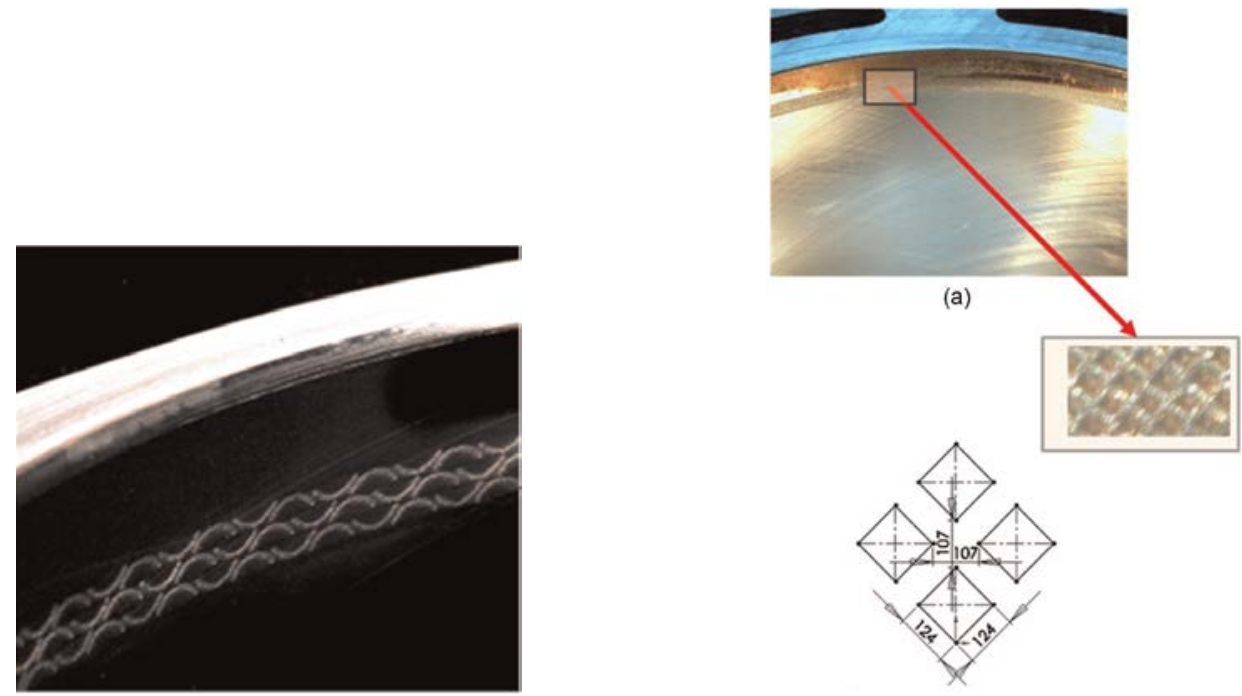

Figure 15.3: Laser textured features produced on a cylinder liner [2]
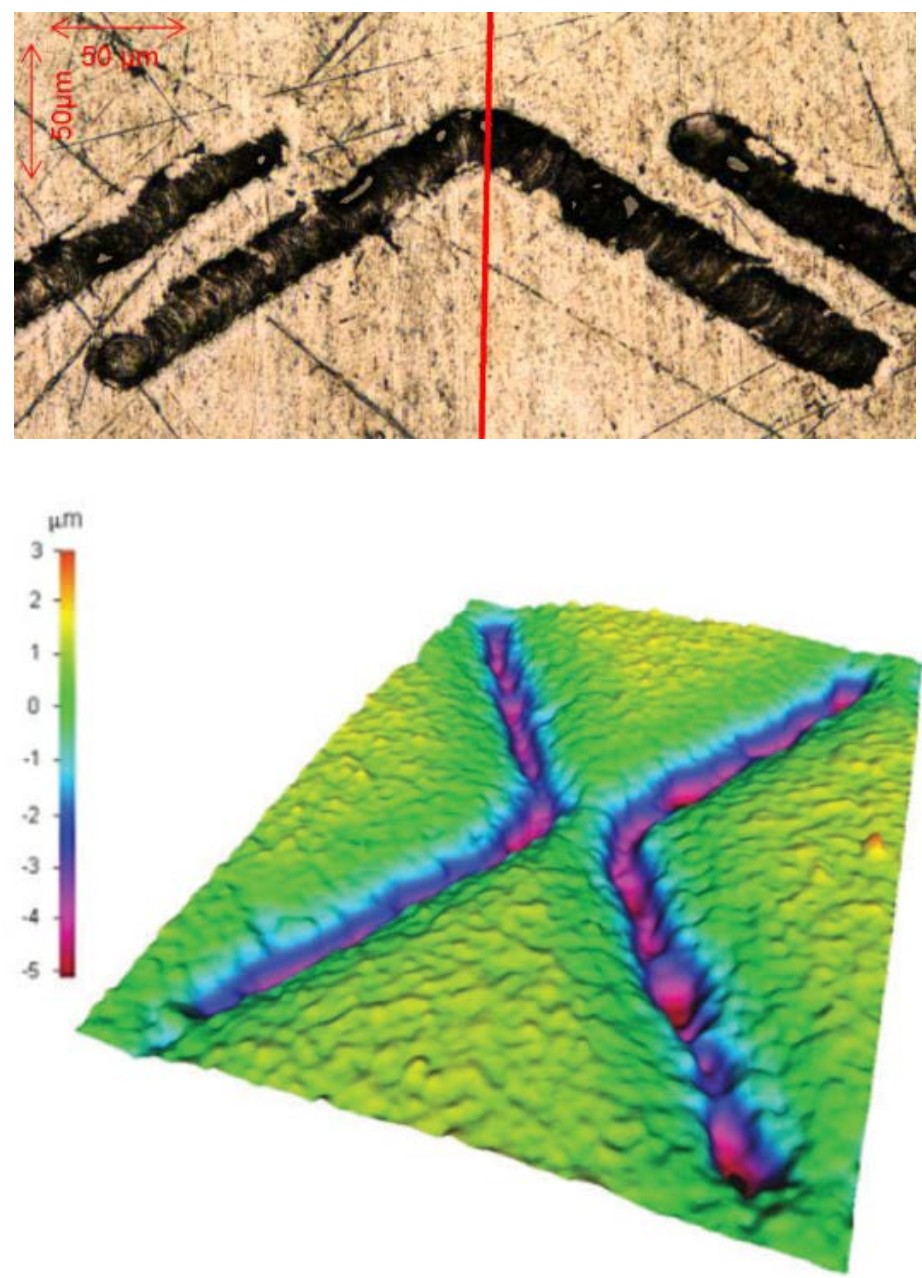

Figure 15.4: Chevron-shaped laser textured features produced on a steel plate [3] 
There are various methods developed to produce textured surfaces. Each method has its own advantages, based on the surface material, level of required precision, quality control and cost of manufacture. Some of the methods comprise various etching techniques, including the use of electrical or photochemical discharge, UV-photo-lithography and laser etching or ablation. Use of abrasive-jet machining, micro-blasting, vibro-rolling, embossing and indentation techniques have also been reported in the literature. Of all these techniques, Laser Surface Texturing (LST) has gained more traction due to its relative cost effectiveness and ease of control and application.

\section{2- Characterisation of textured surfaces}

Figure 15.5 is a schematic representation of a surface textured bearing surface. The parameters used to characterise a textured surface are; size and location of textured area, distribution of the features on the surface, including their axial and lateral intervals (pitch), their cross-sectional shape, depth and profile.

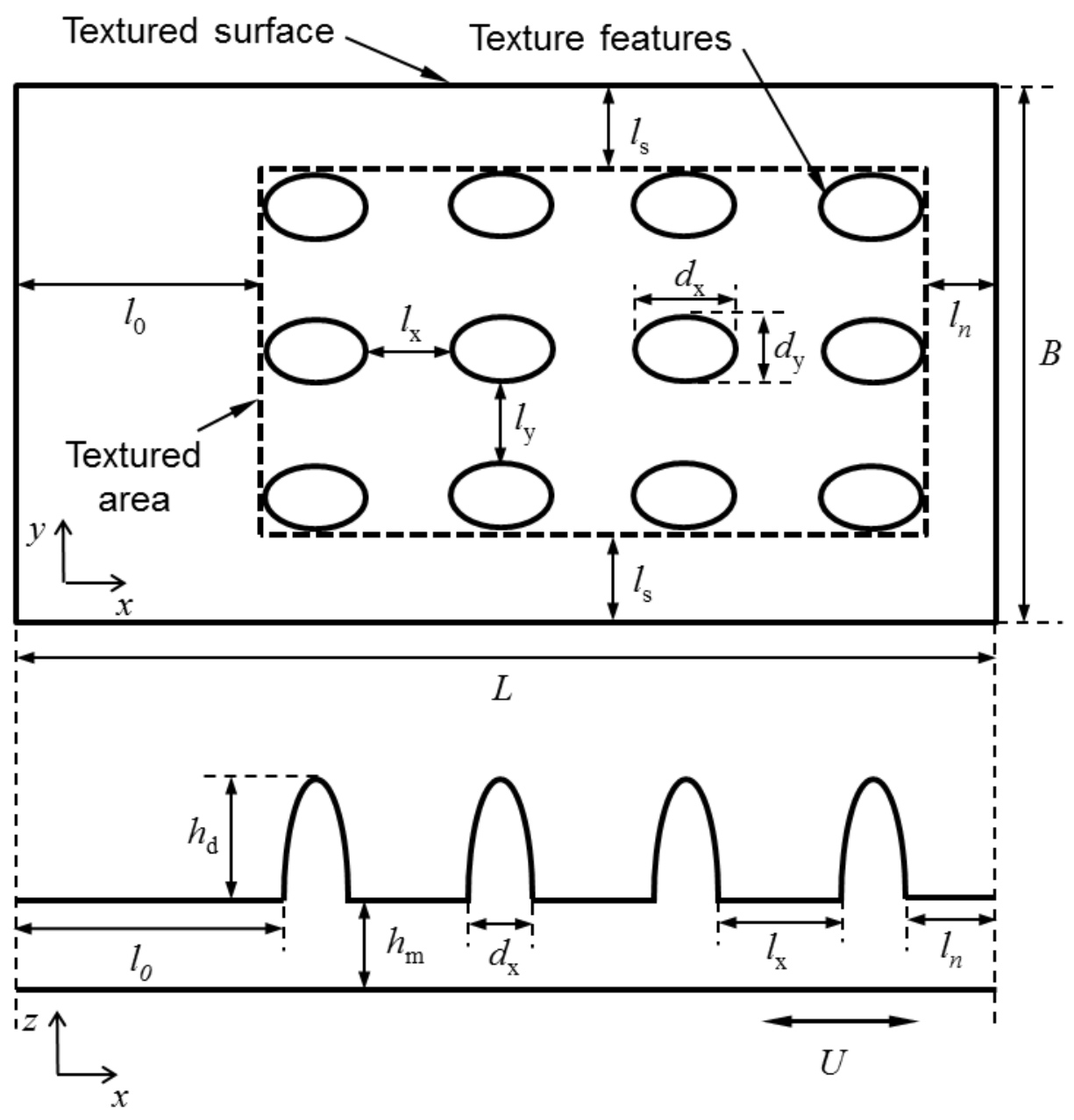

Figure 15.5: Schematics of a textured bearing surface and the corresponding geometrical parameters 
Using the notations in Figure 15.5, the following dimensionless parameters can be defined and used when dealing with textured surfaces [4]:

- Texture depth (or height) ratio: This is the ratio of the maximum to minimum gap between the two contacting surfaces:

$$
\xi=\frac{h_{m}+h_{d}}{h_{m}}=1+\frac{h_{d}}{h_{m}}
$$

- Leading and trailing edge ratios: These are the ratios of leading and trailing edges to the entire bearing axial length:

$\alpha_{0}=\frac{l_{0}}{L}, \alpha_{n}=\frac{l_{n}}{L}$

- The area density of textures: This is the ratio of the projected area of each texture feature to the containing area defined as:

$S_{p}=\frac{A_{d}}{\left(l_{x}+d_{x}\right)\left(l_{y}+y\right)}$

where in the case of dimples (e.g. an elliptical cross-section): $A_{d}=\frac{\pi}{4} d_{x} d_{y}$.

- $\quad$ Textured area ratio: This is the ratio of the textured area to the entire bearing area:

$\kappa=\frac{1}{L B}\left[L-\left(l_{0}+l_{n}\right)\right]\left[B-2 l_{s}\right]$

To model a lubricated contact with a textured surface, Reynolds equation is commonly used. When modelling textured surfaces with finite lateral width features such as those with dimples, pores or chevrons, a two-dimensional form of Reynolds equation should be employed along with numerical solution techniques such as finite difference methods in order to obtain the generated pressure distribution. However, in cases where the lateral width of the texture features can be considered sufficiently long compared with their axial width, then use of a one-dimensional Reynolds equation is justified. In this case analytical solutions may be obtained, which can be used to calculate the tribological performance of the textured contact such as its load carrying capacity and generated viscous friction. This, in turn, would allow for a parametric study of the textured surfaces to find the optimum geometrical parameters for the texture features.

With fully textured contacts and with the possibility of cavitation forming in each texture feature, an analytical solution of Reynolds equation can become a relatively tedious task. However, it is shown that for partially textured conjunctions, where the pressure build-up is based on their cumulative action, it is possible to solve the 1D Reynolds equation analytically. This can be described further using Figures 15.6 and 15.7. 
R. Gohar and H. Rahnejat, Fundamental of Tribology, $3^{\text {rd }}$ Ed., World Scientific Publishing Europe Ltd., Singapore, 2018

Figure 15.6 shows examples of fully and partially textured surfaces. Figure 15.7 shows the generated pressure distribution in fully and partially textured bearings, using rectangular shaped textures. It can be seen that in the fully textured case the pressure inside all the cavities can fall below the atmospheric pressure. Therefore, it is expected that the lubricant film within the cavity would cavitate as the pressure drops below the vaporisation pressure at the bearing operating temperature. Alternatively, the pressure falls to the ambient if the cavity boundaries are exposed to atmosphere.

In the case of partially textured bearings, there is a slight chance of some cavitation at the trailing edge of the texture contact in certain configurations. Figure 15.7 shows that the possibility of cavitation may be reduced by minimising the length of the trailing edge until the effect of cavitation is completely removed, when $l_{n} \approx 0$.

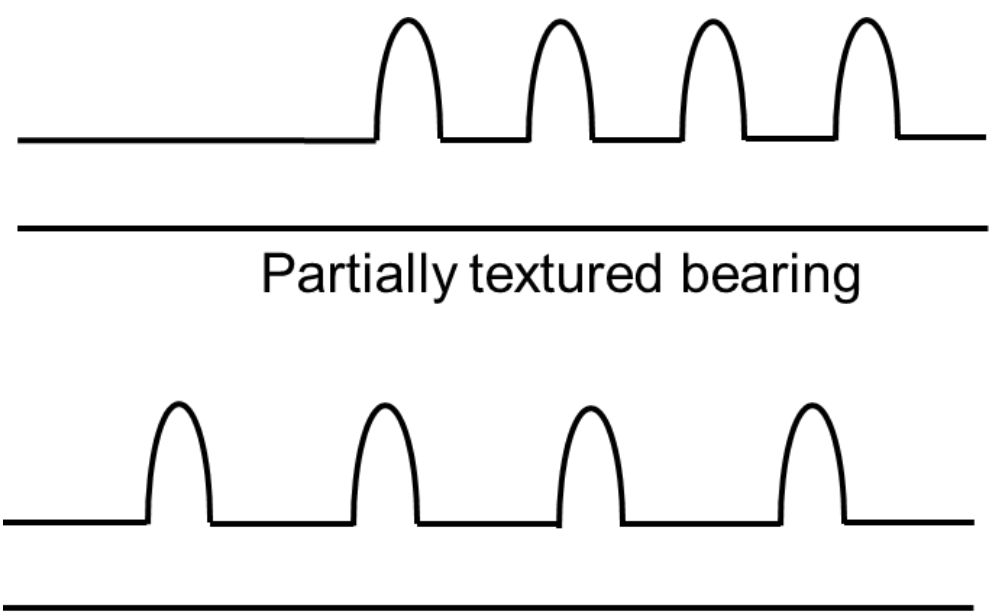

\section{Fully textured bearing}

Figure 15.6: Schematics of partially and fully textured bearings

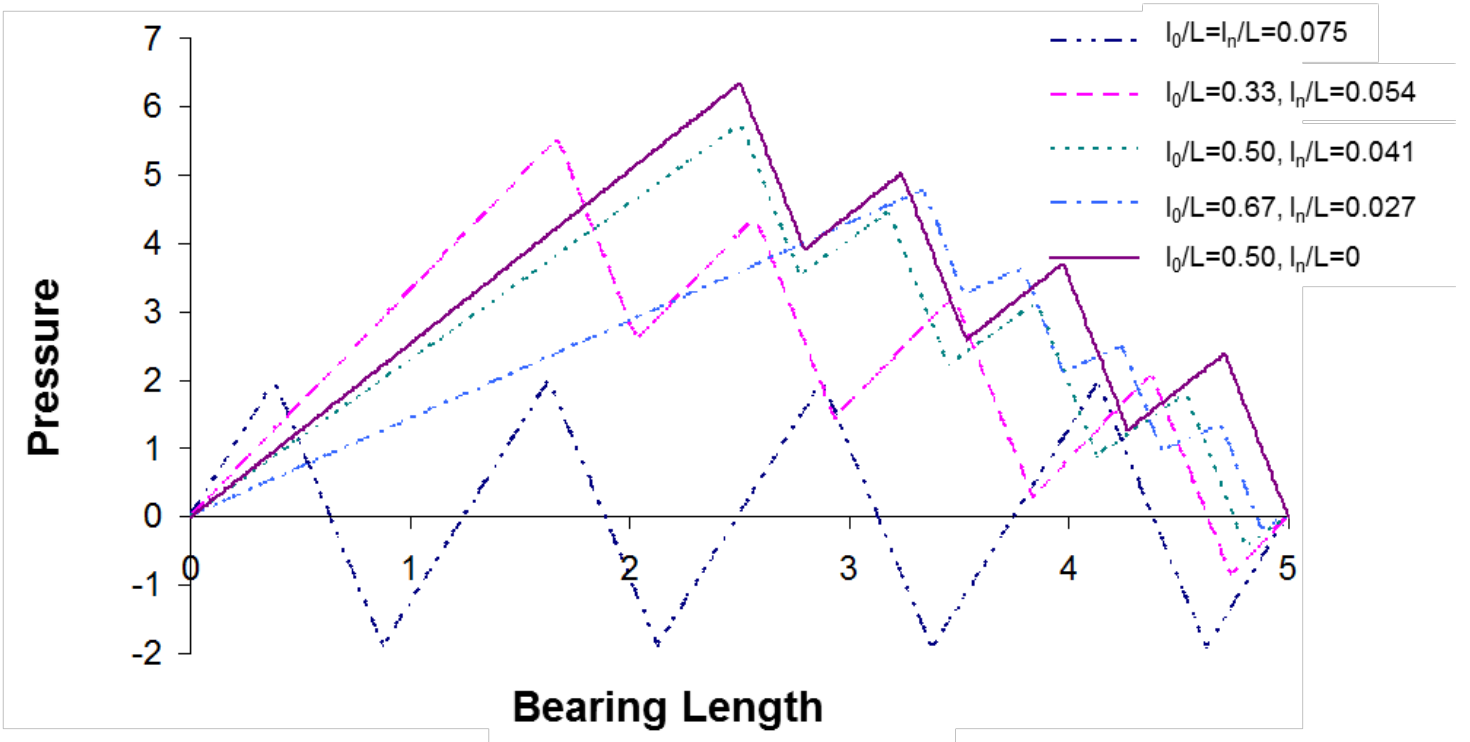

Figure 15.7: Examples of pressure distribution for fully and partially textured sliders with various leading and trailing edge values $\left(l_{0}, l_{n}\right)[4]$ 


\section{3- Performance evaluation of partially textured contacts}

Defining the dimensionless load carrying capacity and friction as:

$\Lambda_{w}=\frac{W h_{m}^{2}}{\eta U L^{2}}, \Lambda_{f}=\frac{f h_{m}}{\eta U L}$

where in a $1 \mathrm{D}$ analysis, $W$ and $f$ are load and friction per unit lateral width of the bearing, $B$, it would be possible to obtain some analytical relations to study the textured surfaces.

For a partially textured bearing with a rectangular or square-shaped texture profile and in the absence of any external hydrostatic pressure at the boundaries with negligible trailing edge length, the dimensionless load carrying capacity, $\Lambda_{W}$ and friction, $\Lambda_{F}$, can be obtained as [5]:

$$
\begin{gathered}
\Lambda_{w}=\frac{3 m \varepsilon \kappa(1-\kappa)(\xi-1)}{(m+\varepsilon-1) \xi^{3}-m \varepsilon \kappa\left(\xi^{3}-1\right)} \\
\Lambda_{f}=\frac{m^{2} \varepsilon^{2} \kappa^{2}(\xi-1)^{4}+\xi(m+\varepsilon-1)\left[(m+\varepsilon-1) \xi^{3}-2 m \varepsilon \kappa(\xi-1)\left(\xi^{2}-\xi+2\right)\right]}{\xi(m+\varepsilon-1)\left[(m+\varepsilon-1) \xi^{3}-m \varepsilon \kappa\left(\xi^{3}-1\right)\right]}
\end{gathered}
$$

where, $m \in[1, \infty)$ is the number of texture features and $\varepsilon$ is one-dimensional equivalent of the $S_{p}$ defined above, as:

$$
\varepsilon=\frac{d_{x}}{l_{x}}
$$

Using the conventional definition of textured surfaces in the form of cavities (negative or concave textures carved into the surface), the range of values for the texture width-to-pitch ratio will be: $\varepsilon \in(0,1]$. If the pitch or distance between features becomes less than the width of the textures (i.e. $\varepsilon>1$ ), then this implies that the textured surface is made of rather protruding features (positive or convex textures). Here we confine ourselves to the former case.

In addition, the textured length ratio, which is the $1 \mathrm{D}$ equivalent of the textured area ratio, $\kappa$, defined above, is given by:

$$
\kappa=1-\left(\alpha_{0}+\alpha_{n}\right)
$$

where, for $\kappa \in(0,1]$, at one extreme there is no textured feature on the surface (i.e. $\kappa=0$ ) and at the other extreme (i.e. $\kappa=1$ ), the contact is considered to be fully textured.

Textures with flat bases (bottom) can be produced using photo-etching methods, whilst using lasers, produces textures usually consisting of rather curved walls, when viewed from the side, approaching a parabolic or triangular shaped profile. If such texture profiles are approximated with an isosceles triangle, then it is possible to obtain corresponding dimensionless relationships for load carrying capacity and friction, in the absence of any external hydrostatic pressure at the boundaries, as [5]: 
R. Gohar and H. Rahnejat, Fundamental of Tribology, $3^{\text {rd }}$ Ed., World Scientific Publishing Europe Ltd., Singapore, 2018

$$
\begin{gathered}
\Lambda_{w}=\frac{3 m \varepsilon \kappa(1-\kappa)(\xi-1)}{2(m+\varepsilon-1) \xi^{2}-m \varepsilon \kappa(2 \xi+1)(\xi-1)} \\
\Lambda_{f}=\frac{4 m \varepsilon \kappa}{(\xi-1)(m+\varepsilon-1)} \ln (\xi)+\frac{2\left\{(m+\varepsilon-1)\left[(m+\varepsilon-1) \xi^{2}-2 m \varepsilon \kappa\left(\xi^{2}+2 \xi-1\right)\right]+m^{2} \varepsilon^{2} \kappa^{2}(\xi+5)(\xi-1)\right\}}{(m+\varepsilon-1)\left[2(m+\varepsilon-1) \xi^{2}-m \varepsilon \kappa\left(2 \xi^{2}-\xi-1\right)\right]}
\end{gathered}
$$

In the relationship for the dimensionless load carrying capacity, it is assumed that the arrangement of textures allows for $\alpha_{n} \approx 0$.

\section{4- Design of textured surfaces for a particular application}

One of the parameters used to characterise textured surfaces, based on the above stated definitions, is the texture height ratio, $\xi$. Specifying the depths of textures requires prediction of the actual minimum film thickness, $h_{m}$, when the surface is in operation. However, this itself is a function of the operating conditions such as the applied load, sliding speed and the operating temperature, which are not usually constant.

\section{Example}

In a sliding contact tribometer, a partially textured $\left(l_{n} \approx 0\right.$ ) flat surface with dimensions of $L \times B=2.2 \times 30 \mathrm{~mm}^{2}$ is desired to run with a speed of $U=10 \mathrm{~mm} / \mathrm{s}$, against a nominally flat steel surface lubricated with an oil with dynamic viscosity of $\eta=0.01$ Pa.s at $\theta=$ $100{ }^{\circ} \mathrm{C}$. The combined root mean square (RMS) roughness of both surfaces is $\sigma_{c}=0.5 \mu \mathrm{m}$. The axial cross-sectional profile of the texture features is approximated triangular with a base length of $d_{x}=50 \mu \mathrm{m}$ and maximum depth of $h_{d}=2 \mu \mathrm{m}$. The edge-to-edge distance (pitch) between the features is given as $l_{x}=125 \mu \mathrm{m}$ and the total number of textures is: $m=10$.

(a) Calculate the maximum load above which the contact may experience a mixed regime of lubrication. What would the friction under this condition?

(b) Calculate the minimum lubricant film thickness for a contact load of $W=50 \mathrm{~N} / \mathrm{m}$ ?

\section{Solution}

Using the given geometrical parameters, the texture length-to-distance ratio is obtained as:

$$
\varepsilon=\frac{d_{x}}{l_{x}}=0.4
$$

In addition, the textured area ratio of the contact becomes:

$$
\kappa=\frac{m d_{x}+(m-1) l_{x}}{L}=0.74
$$


R. Gohar and H. Rahnejat, Fundamental of Tribology, $3^{\text {rd }}$ Ed., World Scientific Publishing Europe Ltd., Singapore, 2018

(a) A mixed regime of lubrication can prevail if the Stribeck film ratio; $\lambda=h_{m} / \sigma_{c}<3$ (chapter 1). Therefore, $h_{m}=1.5 \mu \mathrm{m}$ would be considered as the threshold minimum film thickness required to maintain a hydrodynamic regime of lubrication. If the applied contact load increases and as a result the minimum film thickness reduces, then a mixed regime of lubrication would prevail.

Knowing threshold value for the minimum lubricant film thickness, the expected texture depth (or height) ratio can be calculated as:

$$
\xi=1+\frac{h_{d}}{h_{m}}=2.33
$$

Consequently, the corresponding dimensionless load carrying is obtained as:

$$
\Lambda_{w}=\frac{3 m \varepsilon \kappa(1-\kappa)(\xi-1)}{2(m+\varepsilon-1) \xi-m \varepsilon \kappa(2 \xi+1)(\xi-1)}=0.14
$$

which would result in a load carrying capacity per unit lateral width of:

$$
W=\eta U\left(\frac{L}{h_{m}}\right)^{2} \Lambda_{w}=30.8 \mathrm{~N} / \mathrm{m}
$$

The corresponding dimensionless friction becomes:

$$
\begin{aligned}
& \Lambda_{f}=\frac{4 m \varepsilon \kappa}{(\xi-1)(m+\varepsilon-1)} \ln (\xi) \\
& +\frac{2\left\{(m+\varepsilon-1)\left[(m+\varepsilon-1) \xi^{2}-2 m \varepsilon \kappa\left(\xi^{2}+2 \xi-1\right)\right]+m^{2} \varepsilon^{2} \kappa^{2}(\xi+5)(\xi-1)\right\}}{(m+\varepsilon-1)\left[2(m+\varepsilon-1) \xi^{2}-m \varepsilon \kappa\left(2 \xi^{2}-\xi-1\right)\right]}=0.96
\end{aligned}
$$

and therefore, friction for the contact per unit lateral width would be:

$$
f=\eta U\left(\frac{L}{h_{m}}\right) \Lambda_{f}=0.14 \mathrm{~N} / \mathrm{m}
$$

Producing a viscous coefficient of friction of $\mu=0.0046$.

(b) When the applied load changes, providing that all the geometrical and operational conditions remain the same, the lubricant film thickness alters. Hence, the texture depth ratio would take a different value. The new value can be calculated as follow:

Using the specified value for the applied load, the dimensionless load carrying capacity is obtained as:

$$
\Lambda_{w}=\frac{W h_{m}{ }^{2}}{\eta U L^{2}}=0.23
$$

Therefore, using the relationship for the dimensionless load carrying capacity, the actual texture depth ratio is calculated using: 
R. Gohar and H. Rahnejat, Fundamental of Tribology, $3^{\text {rd }}$ Ed., World Scientific Publishing Europe Ltd., Singapore, 2018

$$
\Lambda_{w}=\frac{3 m \varepsilon \kappa(1-\kappa)(\xi-1)}{2(m+\varepsilon-1) \xi-m \varepsilon \kappa(2 \xi+1)(\xi-1)}
$$

Rearranging the above equation with respect to $\xi$ results in a quadratic form which can then be easily solved with respect to $\xi$, yielding: $\xi=2.78$.

This would, in turn, result in the minimum film thickness of $h_{m}=1.12 \mu \mathrm{m}$. Since the obtained value for the minimum film thickness results in Stribeck film ratio of $\lambda=2.25$; then a mixed regime of lubrication would be expected and hence the effect of surface roughness on both load carrying capacity and friction needs to be taken into account.

\section{5- Optimisation of textured surfaces}

In the above example, the texturing parameters were provided and it was required to specify the subsequent tribological conditions, which would be prevalent if those parameters were implemented. With the help of analytical relationships, it would be possible to examine the possibility of finding optimum texture parameters which would yield the maximum load carrying capacity.

Upon mathematical examination of the given relationships for the load carrying capacity for either of the texture profiles, it is can be seen that the load carrying capacity would reduce rather monotonically with an increasing number of textures, $m$, or any increase with the texture width-to-pitch ratio, $\varepsilon$. However, there would exist specific values for $\kappa$ or $\xi$, based on which a maximum possible value for the load carrying capacity can be achieved. Nevertheless, these values would be a function of number of textures and the pitch ratio, for each texture profile. A detailed set of results for optimum values for partially textured surfaces are given in $[5,6]$.

Table 1 summarises the results of optimisation for a special case, where a relatively large number of textures reside in the contact and the distance between the textures is same as their base length. The results for optimum $\xi$ and $\kappa$ are obtained, based on two different objective functions; (i)- maximisation of load carrying capacity and (ii)- minimisation of friction coefficient.

Table 1: Optimisation results based on the analytical functions for a special case [5]

\begin{tabular}{|c|c|c|c|c|c|c|}
\hline \multirow{2}{*}{$\begin{array}{c}m \rightarrow \infty \\
\varepsilon=1.0\end{array}$} & \multicolumn{3}{|c|}{$\begin{array}{c}\text { Based on maximisation of load } \\
\text { carrying capacity }\end{array}$} & \multicolumn{3}{c|}{$\begin{array}{c}\text { Based on minimisation of friction } \\
\text { coefficient }\end{array}$} \\
\cline { 2 - 7 } & $\xi_{\text {opt }}$ & $\kappa_{\text {opt }}$ & $\left(\Lambda_{w}\right)_{\max }$ & $\xi_{\text {opt }}$ & $\kappa_{\text {opt }}$ & $\left(\Lambda_{f} / \Lambda_{w}\right)_{\min }$ \\
\hline Rectangular & 2.1214 & 0.3034 & 0.1548 & 2.2966 & 0.2127 & 4.7910 \\
\hline Triangular & 2.2470 & 0.3961 & 0.0653 & 2.9310 & 0.2864 & 8.2692 \\
\hline
\end{tabular}


R. Gohar and H. Rahnejat, Fundamental of Tribology, $3^{\text {rd }} \mathrm{Ed}$., World Scientific Publishing Europe Ltd., Singapore, 2018

Clearly for any special case, where the number of textures and their distribution is known the optimum texture depth ratio and textured area ratio can be obtained using the already given analytical relationships above.

\section{6- References}

[1] Morris, N.J., Rahnejat, H. and Rahmani, R., “Tribology of partial pad journal bearings with textured surfaces, 3rd European Conference on Tribology (ECOTRIB), Vienna, Austria, 7-9 June 2011, 6pp

[2] Howell-Smith, S., Rahnejat, H., King, P.D. and Dowson, D., "Reducing in-cylinder parasitic losses through surface modification and coating”, Proc. IMechE, Part D: J.

Automobile Engineering, 228(4), 2014: 391-402

[3] Morris, N., Leighton, M., De la Cruz, M., Rahmani, R., Rahnejat, H. and Howell-Smith, S., "Combined numerical and experimental investigation of the micro-hydrodynamics of chevron-based textured patterns influencing conjunctional friction of sliding contacts”, Proc. IMechE, Part J: J. Engineering Tribology, 229(4), 2015: 316-335

[4] Rahmani, R., An investigation into analysis and optimisation of textured slider bearings with application in piston ring/cylinder liner contact, $\mathrm{PhD}$ Thesis, Anglia Ruskin University, 2008

[5] Rahmani, R., Mirzaee, I., Shirvani, A. and Shirvani, H., “An analytical approach for analysis and optimisation of slider bearings with infinite width parallel textures”, Tribology International, 43(8), 2010: 1551-1565

[6] Rahmani, R. and Rahnejat, H., "Enhanced performance of optimised partially textured load bearing surfaces”, Tribology International, 117, 2018: 272-282 